\title{
Chemical structure of the massive protobinary-forming hot core, W3 $\left(\mathrm{H}_{2} \mathrm{O}\right)$
}

\author{
Y. C. Minh ${ }^{1,2}$ and H.-R. Chen ${ }^{2,3}$ \\ ${ }^{1}$ Korea Astronomy and Space Science Institute, Korea \\ ${ }^{2}$ Academia Sinica, Institute of Astronomy and Astrophysics, Taiwan \\ ${ }^{3}$ National Tsing Hua University, Taiwan \\ email: minh@kasi.re.kr, hchen@phys.nthu.edu.tw
}

\begin{abstract}
The hot molecular core, $\mathrm{W} 3\left(\mathrm{H}_{2} \mathrm{O}\right)$, contains a massive protobinary system that is cocooned by dense gas $\left(\mathrm{n}\left(\mathrm{H}_{2}\right) \sim 10^{7} \mathrm{~cm}^{-3}\right)$, with about $1 \operatorname{arcsec}(\sim 2000$ AU) separation of the binary system. We investigate chemical properties of the gas components around this binary system using the mm-wave transitions of complex molecules, $\mathrm{CH}_{3} \mathrm{CN}, \mathrm{SiO}, \mathrm{HNCO}$, and $\mathrm{CH}_{3} \mathrm{CH}_{2} \mathrm{CN}$, observed with the BIMA array. The two protostellar objects, $\mathrm{A}$ and $\mathrm{C}$ in $\mathrm{W} 3\left(\mathrm{H}_{2} \mathrm{O}\right)$, can be distinguished using chemical properties, suggesting that the source A may be younger than the source $\mathrm{C}$ within the time scale of less than $10^{4}$ years. The hot core around the source $\mathrm{C}$, traced by $\mathrm{CH}_{3} \mathrm{CH}_{2} \mathrm{CN}$ and the $\mathrm{K}=6$ component of $\mathrm{CH}_{3} \mathrm{CN}$, seems to have more time for chemical evolution than the source $\mathrm{A}$. The $\mathrm{SiO}$ emission in this region suggests that there was an influence from the outside of the $\mathrm{W} 3\left(\mathrm{H}_{2} \mathrm{O}\right)$ and $\mathrm{W} 3(\mathrm{OH})$ hot cores. The nitrogen chemistry may be more active in the later stage than the oxygen chemistry, but the chemical evolution of the protostellar envelopes may not be monotonic as previously suggested.
\end{abstract}

Keywords. ISM: abundances, ISM: clouds, ISM: molecules, ISM: individual (W3(H2O)), astrochemistry, molecular processes

A massive-star forming hot core, $\mathrm{W} 3\left(\mathrm{H}_{2} \mathrm{O}\right)$, locates at the $6^{\prime \prime}$ east of the UC HII region $\mathrm{W} 3(\mathrm{OH})$. These objects are embedded in the giant molecular complex $\mathrm{W} 3$ at a distance of about $2 \mathrm{kpc}$. W3 $\left(\mathrm{H}_{2} \mathrm{O}\right)$ contains a protostellar (B0.5-0) binary system (the $\mathrm{A}$ and $\mathrm{C}$ component in continuum) in orbit with each other with a velocity difference $\Delta V_{A-C}=2.81 \mathrm{~km} \mathrm{~s}^{-1}$ (Chen et al. 2006, ApJ, 639, 975; and see references therein). The small angular size (a few arcsecond in diameter) of this hot core requires sub-arcsecond angular resolution to properly resolve the two protostellar objects embedded in the dense gas and dust cocoon of $\mathrm{n}\left(\mathrm{H}_{2}\right) \sim 10^{7} \mathrm{~cm}^{-3}$.

The chemical evolutionary time scale is relatively short, which helps to understand the nature of very complicated high mass star forming cores. We model the structure of this protostellar system using the chemical tracers, $\mathrm{CH}_{3} \mathrm{CN}$, SiO, $\mathrm{HNCO}$, and $\mathrm{CH}_{3} \mathrm{CH}_{2} \mathrm{CN}$ observed with the BIMA array. Chemical differences exist between A and C, probably because of the difference in their chemical evolutionary stages.

In the chemical evolution of hot cores there are, at least, two different formation periods of $\mathrm{N}$-containing species, such as $\mathrm{CH}_{3} \mathrm{CN}$, for the warm extended component and the highly turbulent compact component. The fact that $\mathrm{CH}_{3} \mathrm{CH}_{2} \mathrm{CN}$ exists mainly in source $\mathrm{C}$ suggests that source $\mathrm{C}$ is more chemically evolved than source A. HNCO may trace an expanding shell around the highly turbulent gas of $\mathrm{A}$ and $\mathrm{C}$, where YSOs are embedded. The $\mathrm{SiO}$ emission seems to trace the shocked gas existing outside of these hot cores. 